\title{
Current management of pediatric achalasia
}

\author{
Jun Tashiro, Mikael Petrosyan, Timothy D. Kane
}

Division of Pediatric General \& Thoracic Surgery, Children's National Hospital, Washington, DC, USA

Contributions: (I) Conception and design: J Tashiro, TD Kane; (II) Administrative support: J Tashiro, TD Kane; (III) Provision of study materials or patients: J Tashiro, TD Kane; (IV) Collection and assembly of data: J Tashiro, TD Kane; (V) Data analysis and interpretation: All authors; (VI) Manuscript writing: All authors; (VII) Final approval of manuscript: All authors.

Correspondence to: Timothy D. Kane, MD. Chief, Division of General \& Thoracic Surgery, Children's National Hospital and Professor of Surgery and Pediatrics, George Washington University School of Medicine and Health Sciences, 111 Michigan Ave NW, Washington, DC 20010, USA. Email: TKane@childrensnational.org.

\begin{abstract}
Achalasia is a rare condition affecting esophageal motility in children. In a manner similar to the disease found in the adult population, children experience symptoms of dysphagia, regurgitation, and chest pain due to a failure of relaxation of the lower esophageal sphincter. Standard diagnostic approaches include upper endoscopy and esophageal manometry. New developments in diagnosis include highresolution esophageal manometry and the endoscopic functional lumen imaging probe. Therapies available include endoscopic balloon dilations and botulinum toxin injections into the lower esophageal sphincter, as well as surgical interventions. The Heller myotomy was first described in 1913; since then, there have been many modifications to the procedure to improve outcomes and lower morbidity. Currently, the most commonly performed surgical procedure is the laparoscopic Heller myotomy, in which the sphincter muscle is divided using longitudinal incisions with or without a partial fundoplication procedure. In recent years, per oral endoscopic myotomy (POEM) is gaining support as a viable natural orifice therapy for achalasia. Complications of POEM occur at a relatively low rate, and outcomes following the procedure have been promising. The treatment of end-stage achalasia however, may include partial or total esophagectomy with reconstruction if possible. Future research is focused primarily on increasing the efficacy, and lowering complications, of existing therapeutic modalities.
\end{abstract}

Keywords: Esophageal achalasia; heller myotomy; per oral endoscopic myotomy (POEM)

Received: 09 May 2020; Accepted: 06 July 2020; Published: 25 July 2021.

doi: $10.21037 /$ tgh-20-215

View this article at: http://dx.doi.org/10.21037/tgh-20-215

\section{Introduction}

Achalasia is a pathological condition causing dysphagia, reflux, and regurgitation. The hallmarks of diagnosis include esophageal dysmotility and lack of relaxation of the lower esophageal sphincter (LES). While the condition was described by Sir Thomas Willis in 1672, the term "achalasia" was first published in 1929 (1,2). Its primary pathophysiology is thought to be related to the loss of ganglion cells in the distal esophageal segment, causing a dysmotility resulting in LES dysfunction $(2,3)$. Left untreated, the sequelae can be significant, as the proximal esophageal tissue becomes more compliant as a compensatory measure and entirely non-functional by endstage disease (3).

Heller performed the first esophagocardiomyotomy in $1913(2,4)$. Since then, endoscopic therapies have become available, primarily as an initial or non-surgical trial (5). The minimally invasive approach of the Heller myotomy for adults was described in 1993 by Jorgensen et al. and has since shortened recovery, decreased complications, and improved availability of the procedure compared to its open counterpart (6,7). Most recently, the peroral endoscopic myotomy (POEM) was introduced in 2010, which revolutionized the treatment of this condition, providing a 


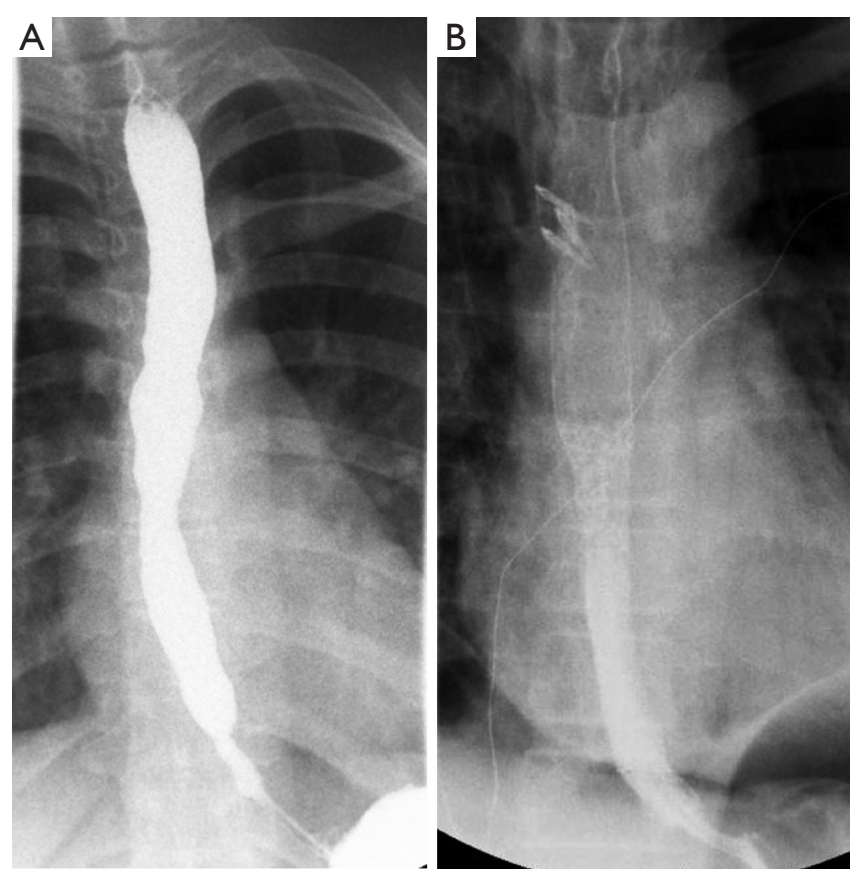

Figure 1 Esophageal achalasia. (A) Pre-operative contrast esophagram demonstrating achalasia. (B) Post-operative contrast esophagram demonstrating successful treatment of achalasia.

viable alternative using a natural orifice approach $(5,8)$.

For the pediatric population, major series of patients being treated for achalasia date back to 1983 (9). As with many pediatric surgical procedures, the introduction of diagnostic and therapeutic modalities has paralleled that for adults, including minimally invasive and endoscopic procedures $(2,5,7)$. New developments, such as advanced esophageal manometry and intraoperative measurement capabilities, can now demonstrate real-time feedback of the effectiveness of the procedure (10).

This article reviews the most current diagnostic and therapeutic modalities available for children, along with outcomes and future directions for research. By understanding the options available for children with this debilitating condition, the authors seek to provide guidance on the most up to date recommendations.

\section{Diagnostic modalities}

Achalasia generally has an insidious onset, with many subclinical features preceding definitive diagnosis. This course is especially true for many children, for whom the diagnosis is made on average at approximately 7 years of age (11). Many patients (46\%) are diagnosed with gastroesophageal reflux disease and treated as such, often for years, prior to a conclusive diagnosis of achalasia (11). A standard evaluation for patients presenting with symptoms consistent with achalasia include endoscopy, an upper gastrointestinal series, and esophageal manometry. A representative upper gastrointestinal series is shown in Figure 1. Whereas the latter two evaluations will often clearly establish the diagnosis, endoscopy will identify reflux related strictures or other esophageal pathology. High-resolution esophageal manometry has significantly improved diagnostic capability, especially when a standardized protocol of pressure parameters is used $(12,13)$. Preoperative manometry readings are demonstrated in Figure 2.

Once the diagnosis is established, the severity of the disease can be quantified objectively. The Eckardt score was developed to catalogue the symptoms of achalasia (14). The scoring system ranges from 0 to 12 , attributing points to four symptoms: dysphagia, regurgitation, chest pain, and weight loss. Comparisons of post-procedural Eckardt scores have been used to evaluate differences between treatment modalities $(15,16)$. A normal Eckardt score is considered to be $£ 3$.

\section{Endoscopic interventions}

Endoscopic interventions for achalasia include endoscopic balloon dilations of the narrowed channel of the sphincter mechanism or direct botulinum toxin injections into the sphincter muscle to relax it. Pneumatic dilation of the LES is an accepted initial treatment modality, particularly for patients who are not optimal surgical candidates, although this rarely applies to children. This procedure has been available as an alternative to surgical intervention for decades, with outcomes in children reported as early as 1983 (9). A relatively low complication rate and wide availability makes this treatment modality attractive as an initial therapy for patients (5). The primary disadvantage however, is that multiple dilations are required for successful relief in about $90 \%$ of patients and long-term results have been inferior to surgical myotomy (5).

Injections of botulinum toxin into the LES directly are another option, especially for children who are poor candidates for repeated pneumatic dilation or surgical intervention $(5,17)$. The toxin serves to provide relaxation of the LES and is also associated with minimal 


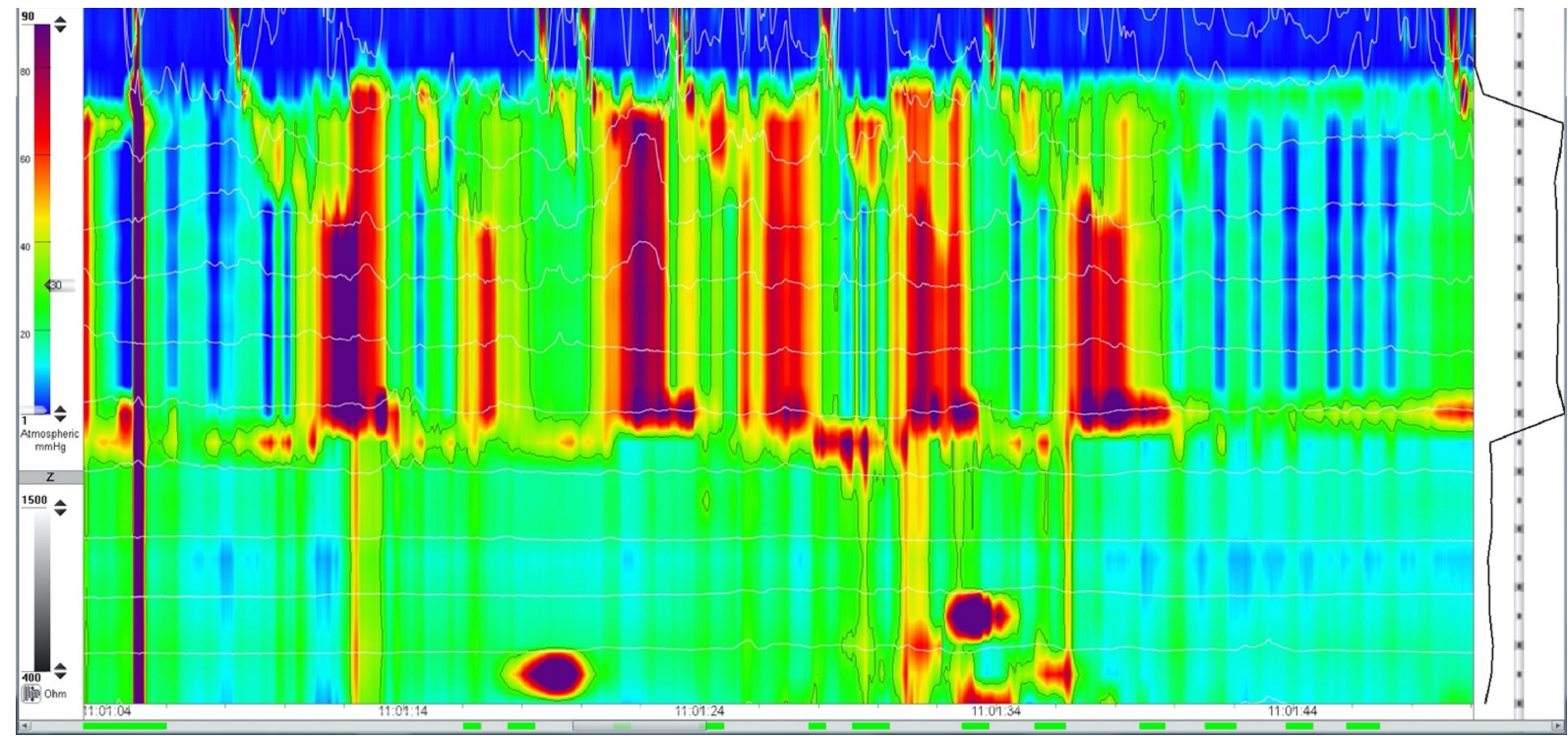

Figure 2 High-resolution esophageal manometry performed in a pre-operative setting, demonstrating a failure of lower esophageal sphincter relaxation.

complications (17). A proven dosing and injection frequency protocols have not been established in the pediatric population (5). Approximately half of patients undergoing this injection require an additional procedure within 7 months (17). In addition, long-term results have also been inferior to surgical myotomy, primarily due to the need for repeated injections for maintenance.

\section{Laparoscopic heller myotomy}

Heller initially described the esophagocardiomyotomy for achalasia in 1913 (4). Berquist et al. first described a series of pediatric patients in 1983, contrasting the effectiveness of pneumatic dilations and Heller myotomy (9). The laparoscopic Heller myotomy (LHM) in children was then published by Holcomb et al. in 1996, in which two pediatric patients were treated successfully (7). Multiple investigations since have demonstrated that LHM is a safe and effective procedure, with the primary benefit of providing a definitive therapy for achalasia (18-23). In comparison to the equivalent open procedure, this approach allows for decreased pain, shorter length of stay, and earlier return to normal activity (22). As a result, LHM has become the standard surgical therapy in children (5). Major steps of the LHM are shown in Figure 3.

The use of fundoplication has been a point of controversy among adult general surgeons performing
LHM (5). Most commonly, a Dor, Toupet, or Nissen fundoplication is performed in children in order to provide anti-reflux benefits (24). According to an International Pediatric Endosurgery Group (IPEG) survey published in 2016, most pediatric surgeons (95\%) tend to perform an anti-reflux procedure at the time of LHM (24). This figure is similar to the case in adults, for whom surgeons perform fundoplications in $86 \%(2,25)$. In our own experience with 37 patients who underwent LHM from ages 9 months to 19 years (mean 12.9 years), we performed Dor fundoplication in $73 \%$ of patients. Patients not receiving a fundoplication (27\%) tended to be younger and more recent cases in our experience.

The advent of intraoperative manometry during LHM allowed for the improvement of success rates in treating pediatric achalasia. This technique was described for children undergoing LHM in 2008 by Jafri et al., with the advantage of real-time feedback on the adequacy and overall effectiveness of the myotomy (26). With preand post-operative measurements of LES pressure, the recurrence of symptoms following LHM were found to be decreased compared to those that did not have intraoperative monitoring (26). As an additional measure to evaluate for the completion of myotomy, we perform an upper endoscopy before and after the procedure to note the difference in caliber at the LES. If manometry is not available at a particular institution, some authors 

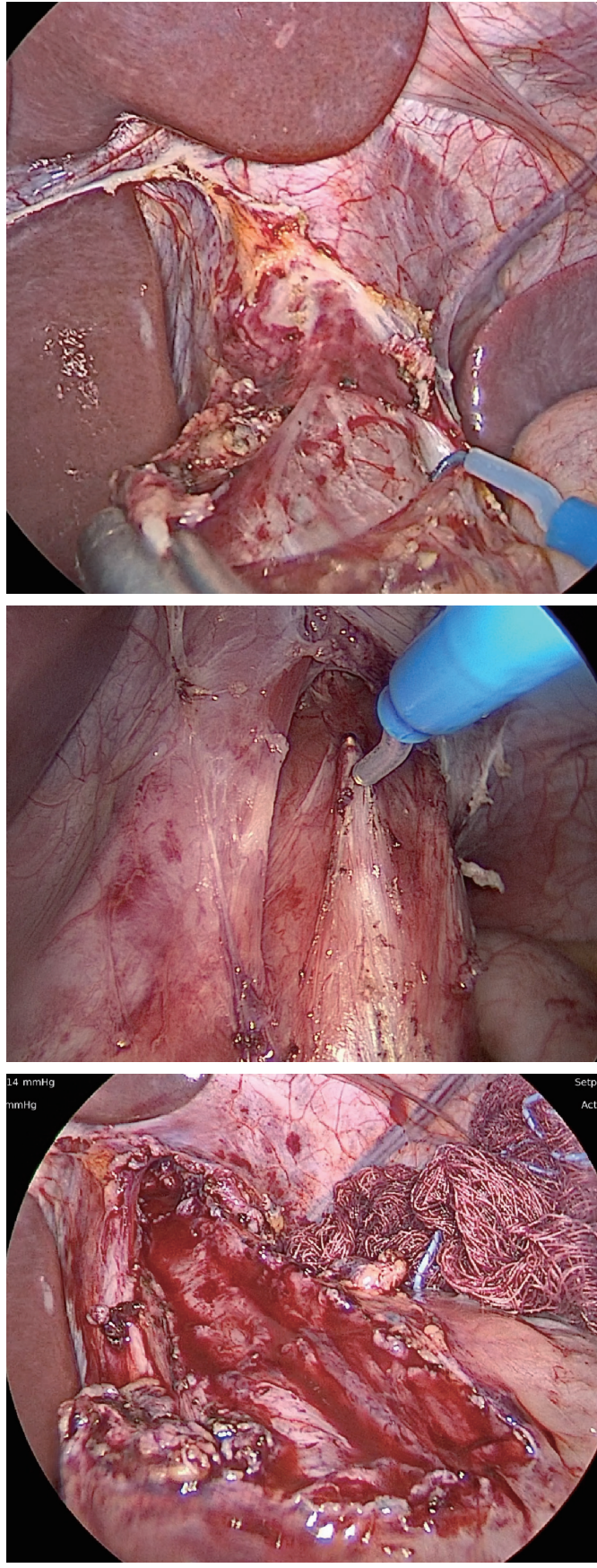

Figure 3 Laparoscopic Heller myotomy. (A) At the start of the case, exposure of the phrenoesophageal ligament is performed using a Nathanson liver retractor. The phrenogastric and gastrohepatic ligaments are incised. (B) A myotomy is performed through the longitudinal and circular fibers. (C) A complete myotomy is carried $3 \mathrm{~cm}$ caudal to the gastroesophageal junction and $6 \mathrm{~cm}$ on the esophagus. have suggested that endoscopy alone may be substituted to evaluate for completion (27).

The use of robotic surgery has been popularized in the adult population, including for the Heller myotomy (28). In the pediatric population however, this is not widely used. In the current literature, only case reports exist and thus the standard remains as the laparoscopic approach (29).

\section{Peroral endoscopic myotomy}

As a natural orifice transluminal endoscopic surgery (NOTES) approach to achalasia, the peroral endoscopic myotomy (POEM) procedure was introduced as an important alternative to LHM. First described by Inoue et al. in 2010, POEM utilizes an endoscope to perform a longitudinal mucosotomy and tunnel down past the gastroesophageal junction (8). After demonstrating its minimally invasive benefit for adult patients, the POEM procedure has become an important treatment for achalasia in children (30). Overall, the clinical success and safety profile appears to be similar to that described in adults $(16,31)$. Major steps of the POEM are depicted in Figure 4.

Outcomes following POEM in children are promising, as long-term follow up has demonstrated lower recurrence rates in comparison to both pneumatic dilation and LHM (32-34). A large, multicenter analysis of outcomes for 117 POEM procedures in children demonstrated that successful resolution of symptoms was achieved in $91 \%$ (31). In addition, a meta-analysis of 146 patients demonstrated a similarly high (93\%) rate of success, with Eckardt scores reduced by an average of nearly 7 points (35). While the procedure is relatively new, the short- and long-term outcomes of the procedure appear to be comparable to LHM with the added benefit of having no incisions (30,31,33,35-37). In a large single-center series, cases of recurrent symptoms requiring additional interventions were fairly high $(16 / 21,76 \%)$; wherein most required only pneumatic dilations (13/21); 2 patients required repeat POEM; and 1 required laparoscopic Heller myotomy to provide definitive symptom relief (37). In our own experience with POEM in children thus far (19 patients), 17 have sustained symptomatic relief $(89.5 \%)$ and 2 have required reintervention. One required a single endoscopic balloon dilatation and the second patient underwent pneumatic dilations and LHM following POEM, amounting to a $10.5 \%$ re-intervention rate. 

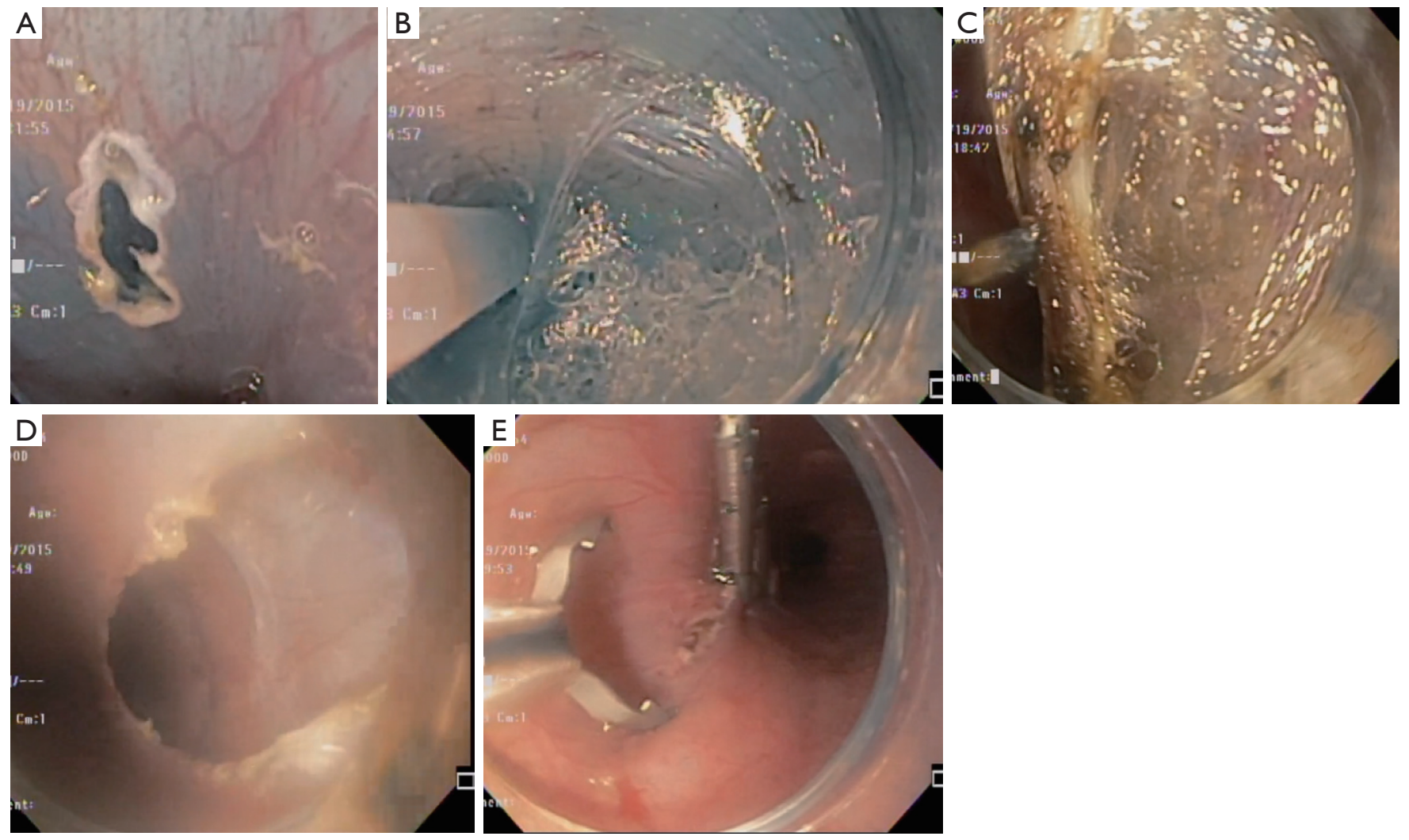

Figure 4 Peroral endoscopic myotomy. (A) A mucosotomy is made and the endoscope is introduced into the submucosal space. (B) A tunnel is extended distally in the submucosal space. (C) The myotomy is performed using an electrocautery blade. (D) The tunnel is visualized following the myotomy. (E) The mucosotomy is closed using endoscopic clips.

Major complications following POEM are relatively low. In adults, pneumothorax $(25 \%)$ and subcutaneous emphysema $(56 \%)$ were most commonly found in a large series (38). In children, Choné et al. found that the incidence of adverse events was relatively low (6\%), with most of these cases classified as mild in severity (31). Other series, including a large, single-center analysis analyzing minimally invasive procedures for pediatric achalasia confirmed that POEM complications remained exceedingly low $(30,35,37)$.

The introduction of the endoscopic functional lumen imaging probe (EndoFLIP, Medtronic) device has allowed for the real-time, intraoperative evaluation of the effectiveness of the procedure $(39,40)$. Similar to intraoperative endoscopic manometry, its use has allowed for the confirmation of an adequate myotomy performed at the time of operation (30). Due to its endoscopic nature, POEM can be easily combined with EndoFLIP to demonstrate adequacy of the myotomy. With these confirmatory measures, POEM remains an important therapeutic modality for pediatric achalasia. An example of intraoperative readings, before and after POEM intervention is demonstrated in Figure 5.

\section{Treatment options for end-stage achalasia}

In patients with end-stage achalasia, partial or total esophageal resection may be required. In these cases, an esophageal reconstruction may be performed using gastric or colon conduits (41). This procedure is associated with a $2 \%$ mortality rate, according to a large meta-analysis in adults; fortunately, end-stage achalasia is an exceedingly rare occurrence in the pediatric population (41).

\section{Future directions}

The development of preventive treatments has been extremely difficult in achalasia, primarily due to the fact that 


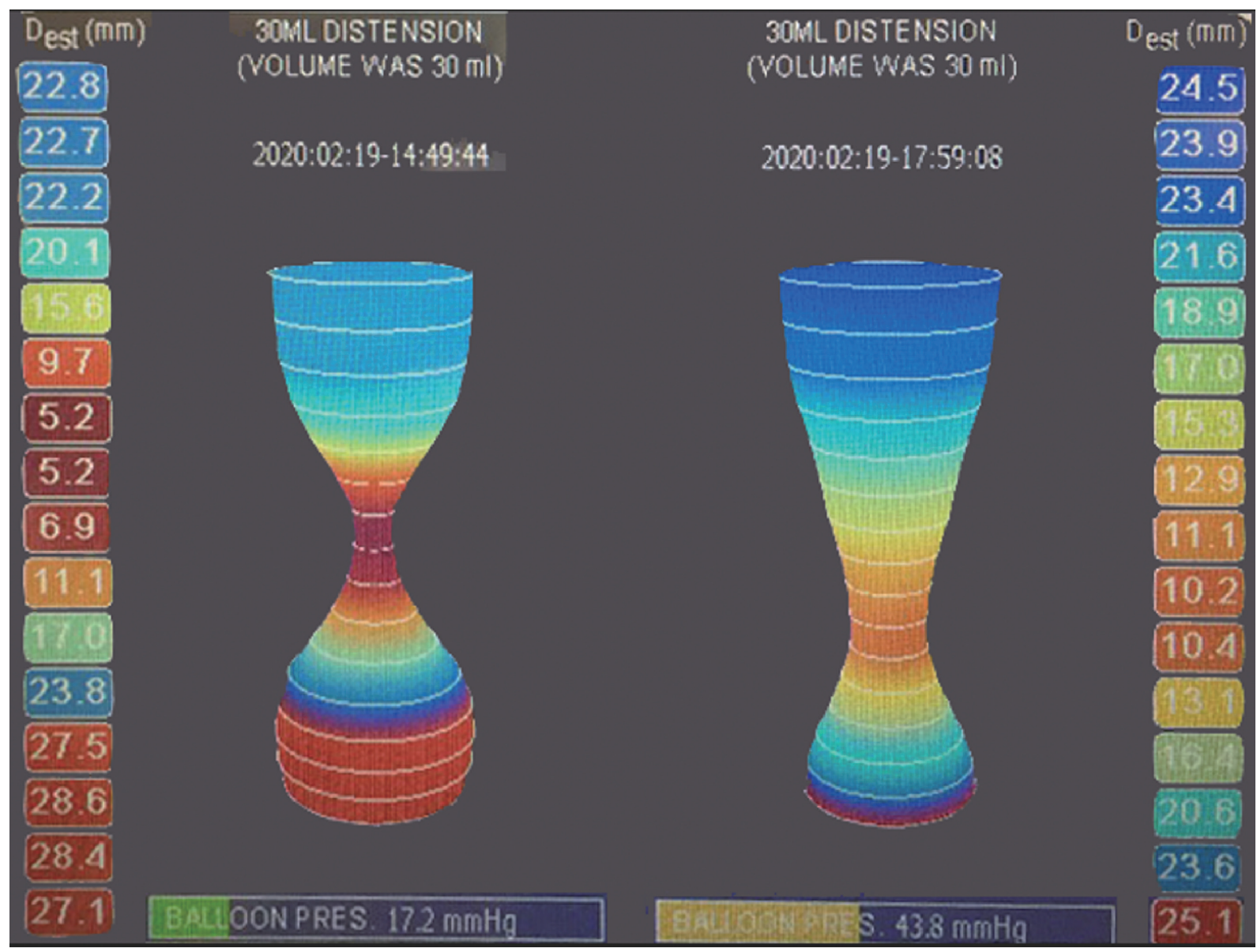

Figure 5 Endoscopic functional lumen imaging probe (EndoFLIP, Medtronic) measurements are shown, prior to (left) and following (right), the per oral endoscopic myotomy (POEM) procedure.

asymptomatic patients cannot be detected (2). Subclinical symptoms may be treated unsuccessfully for years; by the time achalasia is definitively diagnosed, most patients require an intervention - all of which have an observed recurrence rate (16).

Nevertheless, the need for large, prospective trials involving children with achalasia is clear. While metaanalyses and systemic reviews have provided compiled data, the development of an individualized method for determining the best candidates for LHM vs. POEM, along with available endoscopic therapies, remains extremely important for future improvement.

\section{Acknowledgments}

Funding: None.

\section{Footnote}

Provenance and Peer Review: This article was commissioned by the Guest Editors (Eduardo Perez, Samir Pandya, and Matthew S. Clifton) for the series "Current Topics in Pediatric General Surgery" published in Translational Gastroenterology and Hepatology. The article has undergone external peer review.

Conflicts of Interest: All authors have completed the ICMJE uniform disclosure form (available at http://dx.doi. org/10.21037/tgh-20-215). The series "Current Topics in Pediatric General Surgery" was commissioned by the editorial office without any funding or sponsorship. The authors have no other conflicts of interests to declare.

Ethical Statement: The authors are accountable for all 
aspects of the work in ensuring that questions related to the accuracy or integrity of any part of the work are appropriately investigated and resolved.

Open Access Statement: This is an Open Access article distributed in accordance with the Creative Commons Attribution-NonCommercial-NoDerivs 4.0 International License (CC BY-NC-ND 4.0), which permits the noncommercial replication and distribution of the article with the strict proviso that no changes or edits are made and the original work is properly cited (including links to both the formal publication through the relevant DOI and the license). See: https://creativecommons.org/licenses/by-nc-nd/4.0/.

\section{References}

1. Gyawali CP. Achalasia: new perspectives on an old disease. Neurogastroenterol Motil 2016;28:4-11.

2. Islam S. Achalasia. Semin Pediatr Surg 2017;26:116-20.

3. Boeckxstaens GE, Zaninotto G, Richter JE. Achalasia. Lancet 2014;383:83-93.

4. Payne WS. Heller's contribution to the surgical treatment of achalasia of the esophagus. 1914. Ann Thorac Surg 1989;48:876-81.

5. Franklin AL, Petrosyan M, Kane TD. Childhood achalasia: A comprehensive review of disease, diagnosis and therapeutic management. World J Gastrointest Endosc 2014;6:105-11.

6. Jorgensen JO, Hunt DR. Laparoscopic management of pneumatic dilatation resistant achalasia. Aust N Z J Surg 1993;63:386-8.

7. Holcomb GW 3rd, Richards WO, Riedel BD. Laparoscopic esophagomyotomy for achalasia in children. J Pediatr Surg 1996;31:716-8.

8. Inoue $\mathrm{H}$, Minami $\mathrm{H}$, Kobayashi $\mathrm{Y}$, et al. Peroral endoscopic myotomy (POEM) for esophageal achalasia. Endoscopy 2010;42:265-71.

9. Berquist WE, Byrne WJ, Ament ME, et al. Achalasia: diagnosis, management, and clinical course in 16 children. Pediatrics 1983;71:798-805.

10. Yeung F, Wong IYH, Chung PHY, et al. Peroral Endoscopic Myotomy with EndoFLIP and DoubleEndoscope: Novel Techniques for Achalasia in Pediatric Population. J Laparoendosc Adv Surg Tech A 2018;28:343-7.

11. Hallal C, Kieling CO, Nunes DL, et al. Diagnosis, misdiagnosis, and associated diseases of achalasia in children and adolescents: a twelve-year single center experience. Pediatr Surg Int 2012;28:1211-7.

12. Goldani HA, Staiano A, Borrelli O, et al. Pediatric esophageal high-resolution manometry: utility of a standardized protocol and size-adjusted pressure topography parameters. Am J Gastroenterol 2010;105:460-7.

13. Pyun JE, Choi DM, Lee JH, et al. Achalasia Previously Diagnosed as Gastroesophageal Reflux Disease by Relying on Esophageal Impedance-pH Monitoring: Use of HighResolution Esophageal Manometry in Children. Pediatr Gastroenterol Hepatol Nutr 2015;18:55-9.

14. Eckardt VF. Clinical presentations and complications of achalasia. Gastrointest Endosc Clin N Am 2001;11:28192, vi.

15. Caldaro T, Familiari P, Romeo EF, et al. Treatment of esophageal achalasia in children: Today and tomorrow. J Pediatr Surg 2015;50:726-30.

16. Goneidy A, Cory-Wright J, Zhu L, et al. Surgical Management of Esophageal Achalasia in Pediatrics: A Systematic Review. Eur J Pediatr Surg 2020;30:13-20.

17. Hurwitz M, Bahar RJ, Ament ME, et al. Evaluation of the use of botulinum toxin in children with achalasia. J Pediatr Gastroenterol Nutr 2000;30:509-14.

18. Rothenberg SS, Partrick DA, Bealer JF, et al. Evaluation of minimally invasive approaches to achalasia in children. J Pediatr Surg 2001;36:808-10.

19. Miller KA, Holcomb GW 3rd. Laparoscopic adrenalectomy and esophagomyotomy. Semin Pediatr Surg 2002;11:237-44.

20. Askegard-Giesmann JR, Grams JM, Hanna AM, et al. Minimally invasive Heller's myotomy in children: safe and effective. J Pediatr Surg 2009;44:909-11.

21. Lee CW, Kays DW, Chen MK, et al. Outcomes of treatment of childhood achalasia. J Pediatr Surg 2010;45:1173-7.

22. Esposito C, Riccipetitoni G, Chiarenza SF, et al. Longterm results of laparoscopic treatment of esophageal achalasia in children: a multicentric survey. J Laparoendosc Adv Surg Tech A 2013;23:955-9.

23. Zagory JA, Golden JM, Demeter NE, et al. Heller Myotomy Is Superior to Balloon Dilatation or Botulinum Injection in Children with Achalasia: A Two-Center Review. J Laparoendosc Adv Surg Tech A 2016;26:483-7.

24. Gould JL, Rentea RM, St Peter SD. Contemporary Management of Achalasia by Pediatric Surgeons: A Survey of the International Pediatric Endosurgical Group. J Laparoendosc Adv Surg Tech A 2016;26:567-9.

25. Costantino CL, Geller AD, Visenio MR, et al. Outcomes 
of Laparoscopic Heller Myotomy for Achalasia: 22-Year

Experience. J Gastrointest Surg 2020;24:1411-6.

26. Jafri M, Alonso M, Kaul A, et al. Intraoperative manometry during laparoscopic Heller myotomy improves outcome in pediatric achalasia. J Pediatr Surg 2008;43:6670; discussion 70 .

27. Adikibi BT, MacKinlay GA, Munro FD, et al. Intraoperative upper GI endoscopy ensures an adequate laparoscopic Heller's myotomy. J Laparoendosc Adv Surg Tech A 2009;19:687-9.

28. Damani T, Ballantyne G. Robotic Foregut Surgery. Surg Clin North Am 2020;100:249-64.

29. Chaer RA, Jacobsen G, Elli F, et al. Robotic-assisted laparoscopic pediatric Heller's cardiomyotomy: initial case report. J Laparoendosc Adv Surg Tech A 2004;14:270-3.

30. Petrosyan M, Khalafallah AM, Guzzetta PC, et al. Surgical management of esophageal achalasia: Evolution of an institutional approach to minimally invasive repair. J Pediatr Surg 2016;51:1619-22.

31. Choné A, Familiari P, von Rahden B, et al. Multicenter Evaluation of Clinical Efficacy and Safety of Per-oral Endoscopic Myotomy in Children. J Pediatr Gastroenterol Nutr 2019;69:523-7.

32. Tan Y, Zhu H, Li C, et al. Comparison of peroral endoscopic myotomy and endoscopic balloon dilation for primary treatment of pediatric achalasia. J Pediatr Surg 2016;51:1613-8.

33. Kethman WC, Thorson CM, Sinclair TJ, et al. Initial experience with peroral endoscopic myotomy for treatment

doi: $10.21037 / \operatorname{tgh}-20-215$

Cite this article as: Tashiro J, Petrosyan M, Kane TD. Current management of pediatric achalasia. Transl Gastroenterol Hepatol 2021;6:33. of achalasia in children. J Pediatr Surg 2018;53:1532-6.

34. Miao S, Wu J, Lu J, et al. Peroral Endoscopic Myotomy in Children With Achalasia: A Relatively Long-term Singlecenter Study. J Pediatr Gastroenterol Nutr 2018;66:257-62.

35. Lee Y, Brar K, Doumouras AG, et al. Peroral endoscopic myotomy (POEM) for the treatment of pediatric achalasia: a systematic review and meta-analysis. Surg Endosc 2019;33:1710-20.

36. Nabi Z, Ramchandani M, Darisetty S, et al. Impact of prior treatment on long-term outcome of peroral endoscopic myotomy in pediatric achalasia. J Pediatr Surg 2019;S0022-3468(19)30465-8.

37. Wood LS, Chandler JM, Portelli KE, et al. Treating children with achalasia using per-oral endoscopic myotomy (POEM): Twenty-one cases in review. J Pediatr Surg 2020;55:1006-12.

38. Ren Z, Zhong Y, Zhou P, et al. Perioperative management and treatment for complications during and after peroral endoscopic myotomy (POEM) for esophageal achalasia (EA) (data from 119 cases). Surg Endosc 2012;26:3267-72.

39. Kim GH. Is EndoFLIP Useful for Predicting Clinical Outcomes after Peroral Endoscopic Myotomy in Patients with Achalasia? Gut Liver 2019;13:3-4.

40. Chuah SK, Lim CS, Liang CM, et al. Bridging the Gap between Advancements in the Evolution of Diagnosis and Treatment towards Better Outcomes in Achalasia. Biomed Res Int 2019;2019:8549187.

41. Aiolfi A, Asti E, Bonitta G, et al. Esophageal Resection for End-Stage Achalasia. Am Surg 2018;84:506-11. 